\title{
COMMENTARY
}

\section{Preventing severe hypoxia during emergent intubation: is nasopharyngeal oxygenation the answer?}

\author{
Lynn P Roppolo* and Jane G Wigginton² \\ See related research by Engström et al., http://ccforum.com/content/14/3/R93
}

\begin{abstract}
Critically ill patients requiring emergent endotracheal intubation are at risk for life-threatening hypoxemia during the intubation procedure, particularly when the patient is apneic and not receiving any supplemental oxygen. In a current study, Engström and colleagues investigated the effect of nasopharyngeal oxygenation in eight anesthetized pigs with induced acute lung injury. The investigators confirmed, even in this model, that pharyngeal oxygenation significantly prolonged the time to desaturation during periods of apnea. Recognizing the limitations of directly extrapolating these experimental results to critically ill human subjects, the findings do support the contention that, until proven otherwise, nasopharyngeal oxygenation should at least be considered as one technique to diminish hypoxemic complications in very sick patients, particularly those with underlying pulmonary impairment.
\end{abstract}

The prospective investigation by Engström and colleagues in a previous issue of Critical Care sets out to determine if apneic oxygenation via a pharyngeal catheter during endotracheal intubation would prevent or increase the time to life-threatening hypoxemia in an animal model with acute lung injury [1]. In this investigation, eight anesthetized piglets with induced lung collapse were randomized to receive either $10 \mathrm{~L}$ of oxygen per minute via a pharyngeal catheter or no oxygen. The investigators found that pharyngeal oxygenation markedly prolonged

*Correspondence: Lynn.Roppolo@UTSouthwestern.edu

'Emergency Medicine, University of Texas Southwestern Medical Center, 5323 Harry Hines Boulevard, Mail Code 8579, Dallas, TX 75390-8579, USA Full list of author information is available at the end of the article the time to severe systemic arterial oxygen desaturation, defined as oxygen saturation ( $\mathrm{SpO} 2$ ) of less than $60 \%$.

Emergency endotracheal intubation in the critical care unit and in the emergency department can be fraught with complications. Difficulty with oxygenation can be challenging in the critically ill as they have much less reserve and are more prone to desaturate during the brief apneic period that occurs while the patient is being intubated [2]. Traditionally, patients typically do not receive supplemental oxygen as attempts are made to pass the endotracheal tube through the vocal cords. In one prehospital study, for example, it was found that among 54 trauma patients who had been intubated following a rapid sequence induction technique, 57\% experienced significant desaturation with a median duration of 160 seconds [3].

The specific premise of the current investigation by Engström and colleagues was to use an animal model involving induced acute lung injury in order to demonstrate that even in underlying conditions at greater risk for life-threatening hypoxemia, the oxygen desaturation can be minimized by using the pharyngeal catheter supplemental oxygen technique. One limitation of the study was that the authors chose a threshold saturation level that was markedly low (60\%). In other studies of critically ill adults, 'severe hypoxemia' was defined as a $\mathrm{SpO} 2$ of less than $80 \%$ and 'hypoxemia' was defined as a $\mathrm{SpO} 2$ of less than $90 \%[4,5]$. It would be helpful to know when the piglets' $\mathrm{SpO} 2$ fell below $90 \%$, a point at which traditional concerns for hypoxia during intubation typically begin for most clinicians. Though less contentious, the investigators chose a continuous 10-minute period of apnea as their cutoff point for desaturation. One prospective cohort study of endotracheal intubation attempts in critically ill adults demonstrated that $10.3 \%$ of intubations did require 10 or more minutes to achieve [4]. However, these patients had multiple intubation attempts in which they were ventilated with a bag-valvemask (BVM) between intubation attempts. Accordingly, 
it is likely that none of these patients had a continuous 10-minute period of prolonged apnea.

Several reports in the anesthesia literature support the use of nasopharyngeal oxygenation following preoxygenation in human subjects, but all in relatively healthy, cooperative persons. For example, Teller and colleagues [6] conducted a randomized placebo controlled trial in 12 uncomplicated patients undergoing endotracheal intubation during scheduled surgery. An $8 \mathrm{Fr}$ catheter was inserted through a nasal airway and insufflated with oxygen $(3 \mathrm{~L} /$ minute). They found that the arterial oxygen saturation (SaO2) never fell below 97\% during the entire 10 minutes of apnea. How the results of these studies would then translate to critically ill human subjects undergoing emergent intubation remains to be seen, particularly among those with underlying pulmonary impairment. It makes intuitive sense that if one provides supplemental oxygenation using a method that allows simultaneous intubation procedures to take place, the time to desaturation would be prolonged, and the experimental evidence certainly supports that contention. Although the presence of a pharyngeal catheter may provide a barrier to obtaining a good seal when using modern BVM devices, it appears (as observed with previous studies) that such a device could be rapidly replaced whenever the BVM is removed.

Overall, the authors succeeded in demonstrating the benefits of pharyngeal oxygen administration in reducing the risk of desaturation during intubation in the presence of acute lung injury using an animal model. Although the study design implemented in the current investigation would be difficult to repeat in human subjects, this study supports the intuitive contention that pharyngeal oxygenation should be considered during emergent intubation of critically ill patients, particularly during periods of apnea. Severe life-threatening hypoxemia is a wellknown adverse consequence of emergent intubations and any reasonable measure used to minimize this risk is a reasonable conclusion. The authors should be congratulated for taking this first step in the methodical process of proper evidence-based approaches in clinical care of the critically ill.

\section{Abbreviations \\ $\mathrm{BVM}=$ bag-valve-mask; SpO2 = oxygen saturation}

\section{Competing interests}

The authors declare that they have no competing interests.

\section{Author details}

'Emergency Medicine, University of Texas Southwestern Medical Center, 5323 Harry Hines Boulevard, Mail Code 8579, Dallas, TX 75390-8579, USA. 2Emergency Medicine and Department of Clinical Sciences, University of Texas Southwestern Medical Center, 5323 Harry Hines Boulevard, Mail Code 8579,

Dallas, TX 75390-8579, USA

Published: 3 November 2010

\section{References}

1. Engström J, Hedenstierna G, Larsson A: Pharyngeal oxygen administration increases the time to serious desaturation at intubation in acute lunch injury: an experimental study. Crit Care 2010, 14:R93.

2. Benumof JL, Dagg R, Benumof R: Critical hemoglobin desaturation will occur before return of an unparalyzed state following $1 \mathrm{mg} / \mathrm{kg}$ intravenous succinylcholine. Anesthesiology 1997, 87:979-982.

3. Dunford JV, Davis DP, Ochs M, Doney M, Hoyt DB: Incidence of transient hypoxia and pulse rate reactivity during paramedic rapid sequence intubation. Ann Emerg Med 2003, 42:721-728.

4. Griesdale DEG, Bosma TL, Kurth T, Isac G, Chittock DR: Complications of endotracheal intubation in the critically ill. Intensive Care Med 2008, 34:1835-1842.

5. Jaber S, Amraoui J, Lefrant J-Y, Arich C, Cohendy R, Landreau L, Calvet Y, Capdevilla X, Mahamat A, Eledjam JJ: Clinical practice and risk factors for immediate complications of endotracheal intubation in the intensive care unit: A prospective, multiple-center study. Crit Care Med 2006, 34:2355-2361.

6. Teller LE, Alexander CM, Frumin MJ, Gross JB: Pharyngeal insufflations of oxygen prevents arterial desaturation during apnea. Anesthesiology 1988, 69:980-982.

doi:10.1186/cc9197

Cite this article as: Roppolo LP, Wigginton JG: Preventing severe hypoxia during emergent intubation: is nasopharyngeal oxygenation the answer? Critical Care 2010, 14:1005. 